\title{
The impact of diabetes on left ventricular diastolic function in patients with arterial hypertension
}

\author{
Murat Araz, ${ }^{1}$ Aysen Bayrac, ${ }^{2}$ Hilmi Ciftci ${ }^{3}$ \\ ${ }^{1}$ Department of Medical Oncology, Malatya State Hospital, Malatya, Turkey \\ ${ }^{2}$ Department of Internal Medicine, Ersoy Hospital, Istanbul, Turkey \\ ${ }^{3}$ Department of Internal Medicine, Medeniyet University Faculty of Medicine, Istanbul, Turkey
}

\begin{abstract}
OBJECTIVE: The aim of this study is to analyse the impact of diabetes mellitus on the left ventricular diastolic function in patients with arterial hypertension.

METHODS: Between July 2007 and July 2008, we enrolled patients aged $\geq 40$ years who had hypertension with or without type 2 diabetes mellitus and unknown history of coronary artery disease who applied to 7.-8. internal medicine polyclinics of Goztepe Education and Training Hospital. Transthoracic echocardiography was used to assess the diastolic function. If patients with positive treadmill exercise test and/or EF $\leq \% 50$ in transthoracic echocardiography were excluded from the study. A total of 110 patients (males, $n=4238.2 \%$, and females, $n=68$; $61.8 \%)$ with a mean age of $60.78( \pm 10.627)$ years were included in the study. For statistical analysis, SPSS 12.0 program and for the comparison of data chi-square test was used.
\end{abstract}

RESULTS: Diastolic dysfunction was significantly more prevalent in diabetes $(81.25 \%)$ than those without diabetes group $(62.9 \%)(p<0.05)$. In men, $35.9 \%$ in the $\mathrm{DM}(+)$ group and $41.1 \%$ in the DM(-) group had diastolic dysfunction. In women, $64.1 \%$ in the DM(+) group and $58.29 \%$ in the DM(-) group had diastolic dysfunction. In the evaluation based on gender, the difference male and female patients was not significant $(p>0.05)$.

CONCLUSION: Diabetes in association with hypertension has a negative effect on left ventricular diastolic function. This effect appears similar in men and women.

Keywords: Diabetes mellitus; diastolic dysfunction; hypertension.

Leart failure is generally associated with im1 paired left ventricular systolic function. However, $30-40 \%$ of all patients with typical symptoms of congestive heart failure have a nor- mal or minimally reduced ejection fraction. In these patients, diastolic dysfunction is the most important reason if not a primary cause of congestive heart failure $[1,2,3]$. 
Newly onset heart failure is related to male sex, ischemic heart disease and systolic heart failure in patients with younger than 65 years old. In contrast to this data, left ventricular ejection fraction is almost normal in patients older than 70 years with heart failure and diastolic dysfunction is the first detectable abnormality in these patients $[4,5]$.

Diastolic functional abnormalities occurs earlier compared with systolic dysfunction in heart disease because of left ventricular relaxation process is more dependent on energy than ventricular contraction [4].

Diastolic heart failure is a clinical syndrome in patients who had symptoms and signs of heart failure, normal or slightly reduced left ventricular ejection fraction, abnormal left ventricular filling and/ or elevated filling pressures $[3,6]$.

Diastolic dysfunction has a particularly high prevalence in elderly patients and it is generally associated with low mortality but high morbidity. Annual mortality rate of diastolic heart failure is approximately $8 \%$ [3] as compared with annual mortality rate of 15 to $30 \%$ [7] seen in systolic heart failure. Diastolic heart failure is especially seen in the elderly patients with hypertension, valvular heart disease, hypertrophy/restrictive cardiomyopathy, tachycardia and ischemia but it can also occur in a variety of clinical disorders.

Possible mechanisms for diastolic dysfunction include interstitial accumulation of glycoprotein, slow sarcoplasmic calcium reuptake, excessive myocardial fibrosis or altered release of mediators such as nitric oxide and endothelin from a dysfunctional coronary endothelium [8]. The pathophysiology of diastolic dysfunction involves delayed relaxation, impaired left ventricular filling and/or increased stiffness [3].

The gold standard assessment of diastolic function consists of measuring the mean pulmonary capillary wedge pressure and LV end-diastolic pressure by cardiac catheterization $[9,10]$. However, routine use of an invasive procedure for the evaluation of diastolic function is not rational in the polyclinic rooms.

Doppler echocardiography is a simple, non-invasive technique that can be used for the assessment of diastolic function. Transmitral velocity pattern is composed of $\mathrm{E}$ wave (occurring during the rapid filling phase) and lower A wave (arising from atrial contraction) [5]. Transmitral flow pattern rapidly passes through the stages of normal relaxation $(\mathrm{E}>\mathrm{A})$, delayed (impaired) relaxation $(\mathrm{E}<\mathrm{A})$, and restrictive $(\mathrm{E}>>\mathrm{A})$ filling patterns [3].

The researchers have shown that diabetes mellitus results in primary myocardial abnormalities unrelated to ischemic heart disease, hypertension or obesity [8].

Identification of patients with diastolic heart failure is important because these patients almost have a poor prognosis as patients with systolic heart failure. Even asymptomatic patients with diastolic dysfunction are under the increased risk of adverse cardiovascular events [4].

The aim of this study was to analyze the impact of diabetes mellitus (DM) on left ventricular diastolic function in hypertensive patients.

\section{MATERIALS AND METHODS}

Between July 2007 and July 2008, we enrolled patients aged $\geq 40$ years who had hypertension with or without type 2 diabetes mellitus and unknown history of coronary artery disease who applied to $7 .-8$. internal medicine polyclinics of Goztepe Education and Training Hospital. Blood samples and history of coronary angiography and treadmill exercise test results were recorded. Patients with positive treadmill exercise stress test (according to modified Bruce protocol) and/or EF $\leq 50 \%$ in transthoracic echocardiography were excluded from the study. The other exclusion criteria were age $<40$ years, arrhythmias (atrial fibrillation), significant valvular disease, known history of coronary artery disease and chronic renal disease.

Transthoracic echocardiography measurements of diastolic dysfunction were performed according to the American Society of Echocardiography (ASE) guidelines [4]. All echocardiography measurements were performed at midday by the same person according to the American Society of Echocardiography recommendations. Imaging was done while the patient in the left lateral decubitus position. Parasternal short and long axis, apical five 


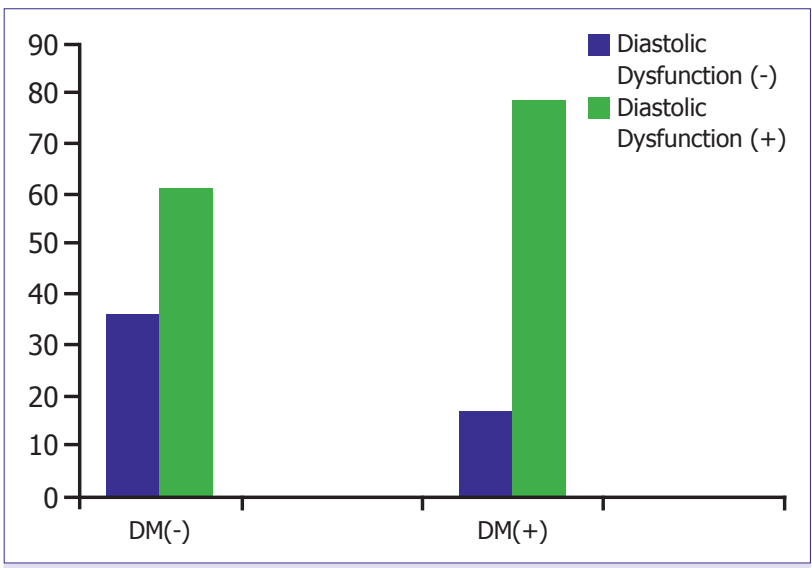

FIGURE 1. Diastolic dysfunction in patients with and without DM.

chamber view was obtained using $\mathrm{M}$ mode, color Doppler and flow Doppler a $2.5 \mathrm{MHz}$ probe of Vivid 3; G.E. pro-brand ultrasound machine. Specifically, transmitral inflow measurements (E/A) were obtained from pulse wave Doppler flow velocity profiles after placing the sample volume at the tip of the mitral valve leaflets. Impaired relaxation was defined as $\mathrm{E} / \mathrm{A}$ ratio $<1$, and an $\mathrm{E} / \mathrm{A}$ ratio between 1 and 2 indicated normal diastolic filling.

Statistical analysis was performed using SPSS (Statistical Package for Social Sciences) 12.0 program. Comparisons of all data were made with chisquare test. P-values were considered significant at a level of $<0: 05$.

The study was conducted in accordance with the Helsinki Declaration and approved by the Local Ethics Committee of Goztepe Education and Training Hospital. All participants gave a written informed consent.

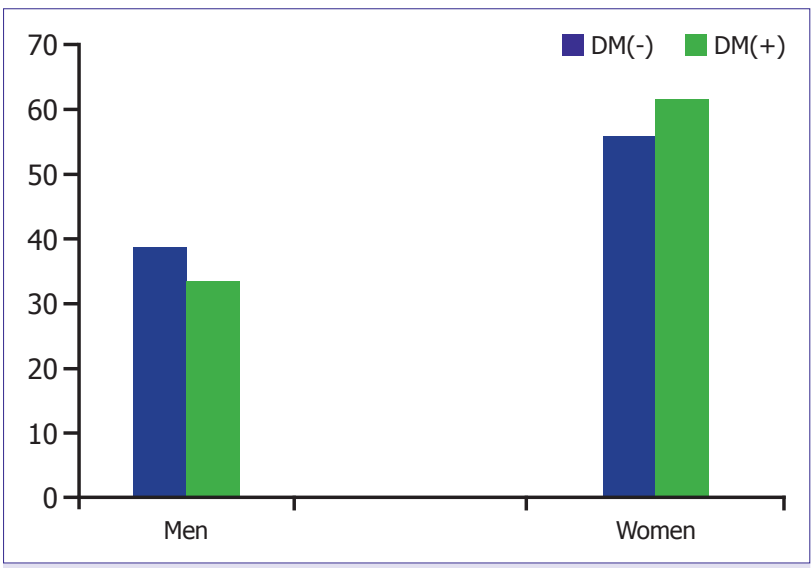

FIGURE 2. Diastolic dysfunction in male and female patients.

\section{RESULTS}

A total of 110 patients (males, $\mathrm{n}=4238.2 \%$, and females, $n=68 ; 61.8 \%$ ) with a mean age of 60.78 $( \pm 10.627)$ years were included in the study. Sixtytwo patients $(56.4 \% ; 25$ male and 37 female) had hypertension without diabetes mellitus and $48 \mathrm{pa}$ tients (43.6\%; 17 male and 31 female) had both hypertension and diabetes mellitus. Mean age of the patients was $60.78 \pm 10.63$ years.

Diastolic dysfunction was significantly more prevalent in patients with DM (81.3\%) than in patients without DM $(62.9 \%)(\mathrm{p}<0.05)$ (Figure 1).

Among men, $35.9 \%$ of the patients with DM (14 patients) and $41.1 \%$ of the patients (16 patients) without DM had diastolic dysfunction. Additionally, $64.1 \%$ of the women with DM (25 patients) and $58.3 \%$ of the women without DM (23 patients) had diastolic dysfunction (Table 1).

TABLE1. Results of diastolic function by gender in diabetic and non-diabetic groups

\begin{tabular}{lccccc} 
& \multicolumn{2}{c}{ Patients with diabetes mellitus plus hypertension } & & \multicolumn{2}{c}{ Patients with only hypertension } \\
\cline { 2 - 3 } E/A ratio & E/A $<1$ & E/A $>1$ & E/A $<1$ & E/A $>1$ \\
& Diastolic dysfunction (+) & Diastolic dysfunction (-) & & Diastolic dysfunction (+) & Diastolic dysfunction (-) \\
\hline Male & 14 & 3 & 16 & 9 \\
Female & 25 & 6 & 23 & 14
\end{tabular}


The prevalence of diastolic dysfunction was not significantly different between men and wome ( $>0.05)$ (Figure 2).

\section{DISCUSSION}

Diabetic cardiomyopathy has been defined in four patients with congestive heart failure by Rubler at al. [11] in the year 1972. These four patients had not hypertension, coronary artery disease, valvular disease and any other reason of cardiomyopathy. Microangiopathic influence and metabolic abnormalities are accepted causes of these abnormalities. Different investigators have shown left ventricular diastolic dysfunction in diabetic patients without clinical manifestations of congestive heart failure [12].

In the absence of risk factors (such as left ventricular hypertrophy, arterial hypertension, coronary artery disease, obesity and diabetes mellitus) diastolic dysfunction and diastolic abnormalities are rare even in elderly patients. According to a study on the absence of risk factors in a group of 1274 people aged $25-75$ years, diastolic abnormalities and dysfunction were seen in $4.3 \%$ and $1.1 \%$ of the patients, respectively. Diastolic abnormalities and diastolic dysfunction were observed in $4.6 \%$, and $1.2 \%$. of the patients over 50 years of age. In the same study, diastolic abnormalities were found to be significantly higher in men than women $(13.8$, and $8.6 \%$, respectively) [13].

In the presence of risk factors, its incidence rise significantly. The rate of was found as $60 \%$ [14] Diastolic dysfunction was found in 14 (60\%) out of 46 asymptomatic type 2 diabetes individuals under glycemic control with non-diabetic complications.

Recently, in a prospective study performed by Watcher et al. the authors investigated the effect of diabetes on left ventricular diastolic function in hypertensive patients. In this study, totally 439 patients were divided into two groups as 315 hypertensive and 124 hypertensive diabetic patients. Diastolic dysfunction was detected at a higher rate in diabetics $(80.6 \%)$ when compared with non-diabetic patients (69.2\%). Moreover, severity of diastolic dysfunction was found to be more serious in patients with diabetes. Sex-specific analysis revealed effects of diabetes on diastolic function in patients with concomitant hypertension. Diastolic dysfunction was determined to be significantly higher in male $(p<0.003)$ patients without any statistically significant difference between genders [15].

In another study, the similar negative effect of diabetes on left ventricular filling pattern had been determined in normotensive and hypertensive individuals. The more serious effect of the combination of diabetes and hypertension was seen on left ventricular relaxation [16]. Poor glycemic control was associated independently with abnormal left ventricular relaxation in this study. In addition, in another study high fasting hyperglycemia was associated with increased myocardial stiffness in hypertensive patients [17].

The impact of diabetes on cardiac function is different between men and women. As a generally accepted corollary male heart seems to be more sensitive to risk factors than female $[15,18,19]$.

According to our study that investigated the effects of diabetes on hypertensive patients, left ventricular diastolic dysfunction was significantly higher in patients with diabetes consistent with the other studies. There was no statistically significant difference between men and women versus other studies which analysed gender differences.

In our study, only E/A ratio was used to determine the diastolic dysfunction. Whereas, American Society of Echocardiography suggested that the different parameters need to be evaluated. Such as the $\mathrm{E} / \mathrm{A}$ ratio, E', E/E'(ratio of early mitral flow velocity (E) to early mitral annulus velocity $\left(E^{\prime}\right)\left(E / E^{\prime}\right)$, and LAVI (left atrial volume index) have been used to estimate the LV filling pressure. There is still a controversy on which parameter, such as E/E' or LAVI, provides a better predictive value for diastolic dysfunction and which parameter is a more powerful prognostic factor for clinical outcomes $[9,10]$.

In our study design, treadmill exercise stress test was used to exclude coronary ischemia. However, this stress test has been limited to detect only coronary ischemia because of the sensitivity level is not very high (approximately 70\%). Myocardial scintigraphy or coronary angiography can be less restric- 
tive invasive methods for further studies to assess coronary ischemia that is a well-known risk factor for diastolic dysfunction.

Good glycemic control in diabetics can help in maintaining normal systolic function. It was shown in the Strong Heart Study that the degree of glycemic control quality is associated with echocardiographic evidence of diastolic function [16]. In our study, glycemic control markers HbA1c and fasting glycemia levels were excluded from evaluation.

Based on multivariate analysis of Strong Heart Study work tasks, ACE inhibitors and AT2 receptor blockers were found to have positive effects on diastolic dysfunction but beta blockers and diuretics did not demonstrate such favourable effects [16]. Therefore, considering the effects of drugs will reduce the limitations of the studies for assessing diastolic dysfunction.

\section{Conclusion}

The prevalence of diastolic dysfunction is higher in hypertensive patients with diabetes mellitus than solely hypertensive patients. Its increased prevalence did not differ between male and female patients.

Conflict of Interest: No conflict of interest was declared by the authors.

Financial Disclosure: The authors declared that this study has received no financial support.

\section{REFERENCES}

1. Bonow RO, Udelson JE. Left ventricular diastolic dysfunction as a cause of congestive heart failure. Mechanisms and management. Ann Intern Med 1992;117:502-10.

2. Cregler LL, Georgiou D, Sosa I. Left ventricular diastolic dysfunction in patients with congestive heart failure. J Natl Med Assoc 1991;83:49-52.

3. Mandinov L, Eberli FR, Seiler C, Hess OM. Diastolic heart failure. Cardiovasc Res 2000;45:813-25.

4. Christopher PA. Diastolic heart function. In: Murphy JG, Lloyd MA (eds), Mayo Clinic Cardiology Concise Textbook (third ed.) Mayo Clinic Scientific Pres 2008:1087-8.

5. Vlahović A, Popović AD. Evaluation of left ventricular diastolic function using Doppler echocardiography. [Article in Croatian] Med Pregl 1999;52:13-8. [Abstract]
6. Aurigemma GP, Gaasch WH. Clinical practice. Diastolic heart failure. N Engl J Med 2004;351:1097-105.

7. Brogan WC 3rd, Hillis LD, Flores ED, Lange RA. The natural history of isolated left ventricular diastolic dysfunction. Am J Med 1992;92:627-30.

8. How to diagnose diastolic heart failure. European Study Group on Diastolic Heart Failure. Eur Heart J 1998;19:990-1003.

9. Han JH, Han JS, Kim EJ, Doh FM, Koo HM, Kim CH, et al. Diastolic dysfunction is an independent predictor of cardiovascular events in incident dialysis patients with preserved systolic function. PLoS One 2015;10:e0118694.

10. Nagueh SF, Appleton CP, Gillebert TC, Marino PN, Oh JK, Smiseth OA, et al. Recommendations for the evaluation of left ventricular diastolic function by echocardiography. Eur J Echocardiogr 2009;10:165-93.

11. Rubler S, Dlugash J, Yuceoglu YZ, Kumral T, Branwood AW, Grishman A. New type of cardiomyopathy associated with diabetic glomerulosclerosis. Am J Cardiol 1972;30:595-602.

12. Shapiro LM, Howat AP, Calter MM. Left ventricular function in diabetes mellitus. I: Methodology, and prevalence and spectrum of abnormalities. Br Heart J 1981;45:122,8.

13. Fischer M, Baessler A, Hense HW, Hengstenberg C, Muscholl $\mathrm{M}$, Holmer S, et al. Prevalence of left ventricular diastolic dysfunction in the community. Results from a Doppler echocardiographic-based survey of a population sample. Eur Heart J 2003;24:320-8.

14. Poirier P, Bogaty P, Garneau C, Marois L, Dumesnil JG. Diastolic dysfunction in normotensive men with well-controlled type 2 diabetes: importance of maneuvers in echocardiographic screening for preclinical diabetic cardiomyopathy. Diabetes Care 2001;24:5-10.

15. Wachter R, Lüers C, Kleta S, Griebel K, Herrmann-Lingen C, Binder L, et al. Impact of diabetes on left ventricular diastolic function in patients with arterial hypertension. Eur J Heart Fail 2007;9:469-76.

16. Liu JE, Palmieri V, Roman MJ, Bella JN, Fabsitz R, Howard BV, et al. The impact of diabetes on left ventricular filling pattern in normotensive and hypertensive adults: the Strong Heart Study. J Am Coll Cardiol 2001;37:1943-9.

17. Jain A, Avendano G, Dharamsey S, Dasmahapatra A, Agarwal $\mathrm{R}$, Reddi $\mathrm{A}$, et al. Left ventricular diastolic function in hypertension and role of plasma glucose and insulin. Comparison with diabetic heart. Circulation 1996;93:1396-402.

18. Rutter MK, Parise H, Benjamin EJ, Levy D, Larson MG, Meigs $\mathrm{JB}$, et al. Impact of glucose intolerance and insulin resistance on cardiac structure and function: sex-related differences in the Framingham Heart Study. Circulation 2003;107:448-54.

19. Du XJ, Samuel CS, Gao XM, Zhao L, Parry LJ, Tregear GW. Increased myocardial collagen and ventricular diastolic dysfunction in relaxin deficient mice: a gender-specific phenotype. Cardiovasc Res 2003;57:395-404. 